\title{
A Trifunctional Self-Immolative Spacer Enables Drug Release with Two Non-Sequential Enzymatic Cleavages
}

\author{
Alberto Dal Corso, ${ }^{* a}$ Simone Arosio, ${ }^{\text {a }}$ Noemi Arrighetti, ${ }^{\text {b }}$ Paola Perego, ${ }^{\mathrm{b}}$ Laura Belvisi, ${ }^{\text {a }}$ Luca \\ Pignataro, a and Cesare Gennari*a
}

Cyclative cleavage of an amine-carbamate self-immolative spacer to deliver a hydroxyl cargo was inhibited by the spacer derivatisation with a phosphate monoester handle. This trifunctional spacer was installed in a model anticancer prodrug which showed a fast drug release only when incubated with both a protease and a phosphatase enzymes.

Self-immolative (SI) spacers are covalent constructs designed to degrade spontaneously in response to specific stimuli. ${ }^{1}$ This disassembly takes place through a variety of intramolecular reactions (mainly electronic cascade in aromatic and $\pi$ extended systems ${ }^{2}$ or cyclization of nucleophilic groups), ${ }^{3}$ resulting in the release of thermodynamically-stable endproducts. Installed in different types of stimuli-responsive materials, SI spacers have found widespread applications, from synthetic and analytical chemistry, ${ }^{4}$ to material sciences ${ }^{5}$ and drug release platforms. ${ }^{6}$ Besides traditional release systems (Figure $1 \mathrm{~A}$ ), in which bifunctional $\mathrm{SI}$ spacers are designed to deliver a single cargo (e.g. a reactive functional group, a dye, a drug), dendrimeric SI spacers have also been designed to simultaneously liberate multiple cargos upon a single chemical trigger (see "signal amplification" in Figure 1B). ${ }^{7}$ On the contrary, the design of SI spacers capable of releasing a single cargo in response to more than one activation signal is not trivial. In particular, chemical strategies for dual-controlled release are gaining momentum in the context of anticancer prodrugs and conjugates, where the tumour-selective activation of highly cytotoxic payloads is fundamental to limit side effects. ${ }^{8}$ So far, two main strategies for dual-controlled cargo release have been proposed: $i$ ) the functionalization of two anchoring points at the cargo's structure with two different triggers (see "Dual Cargo Functionalization" in Figure 1C), ${ }^{9}$ and ii) the functionalization of a single reactive group in the cargo's structure with two triggers connected in series (see "Sequential Activation" in Figure 1C). The latter strategy has been successfully applied to the release of anticancer drugs in response to different combinations of activation sequences. ${ }^{10}$
A) Traditional Release System [Ref 4-6] B) Signal Amplification [Ref 7]

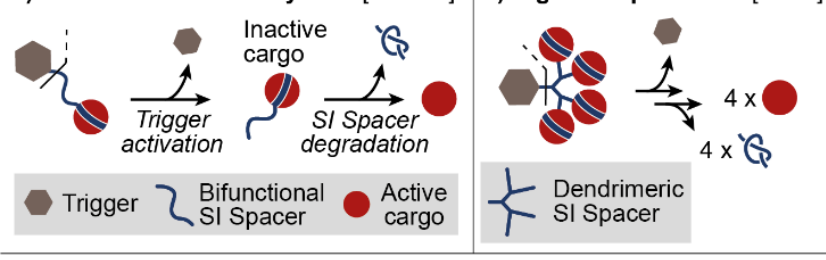

C) Dual Activation

Dual Cargo Functionalization [Ref 9] Sequential Activation [Ref 10]
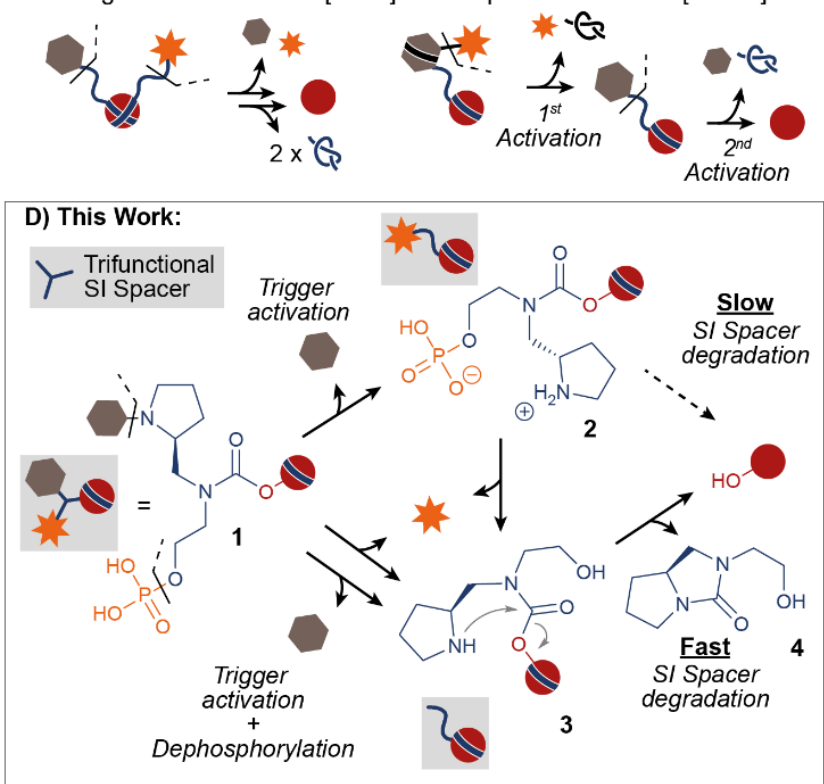

Figure 1. Schematic representation of common strategies to release molecular cargos (e.g. dyes, drugs, etc.) from covalent constructs upon (A) single trigger activation and subsequent degradation of a self-immolative (SI) spacer to deliver a single cargo unit; (B) trigger activation to release multiple cargo units through degradation of a dendrimeric SI spacer; (C) activation of two triggers connected either at two different sites of a single cargo unit (left) or in series (right). D) General structure (1) and dual-activation mechanism of the trifunctional $\mathrm{SI}$ spacer described in this work.

Interestingly, examples of SI spacers prone to non-sequential dual activation by means of two independent stimuli (see Figure 1D) have never been described. Our group recently studied the reactivity of bifunctional amine-carbamate $\mathrm{SI}$ spacers towards cyclative cleavage and release of $\mathrm{OH}$-bearing drugs. In particular, a pyrrolidine-carbamate SI spacer showed the highest cyclization rates, and this feature resulted in enhanced anticancer effects in vitro. ${ }^{11}$ Since the reactivity of amine nucleophiles is generally inhibited under acidic $\mathrm{pH}$, we envisioned that the cargo release could be further modulated by amine-carbamate SI spacer conjugation to a suitable 
Brønsted acid through a cleavable bond, resulting in a trifunctional SI spacer (see general structure 1 in Figure 1D). Ideally, the only trigger activation in $\mathbf{1}$ may not be sufficient to release the cargo, as the amine would be engaged in a zwitterion with the proximal Brønsted acid (structure 2). On the other hand, the fast-cyclizing SI spacer $\mathbf{3}$ may be generated upon two independent and non-sequential events (i.e. main trigger activation and Brønsted acid removal) which release the cyclic urea 4 and the OH-bearing cargo, depicting an unprecedented dual-activation system.

A
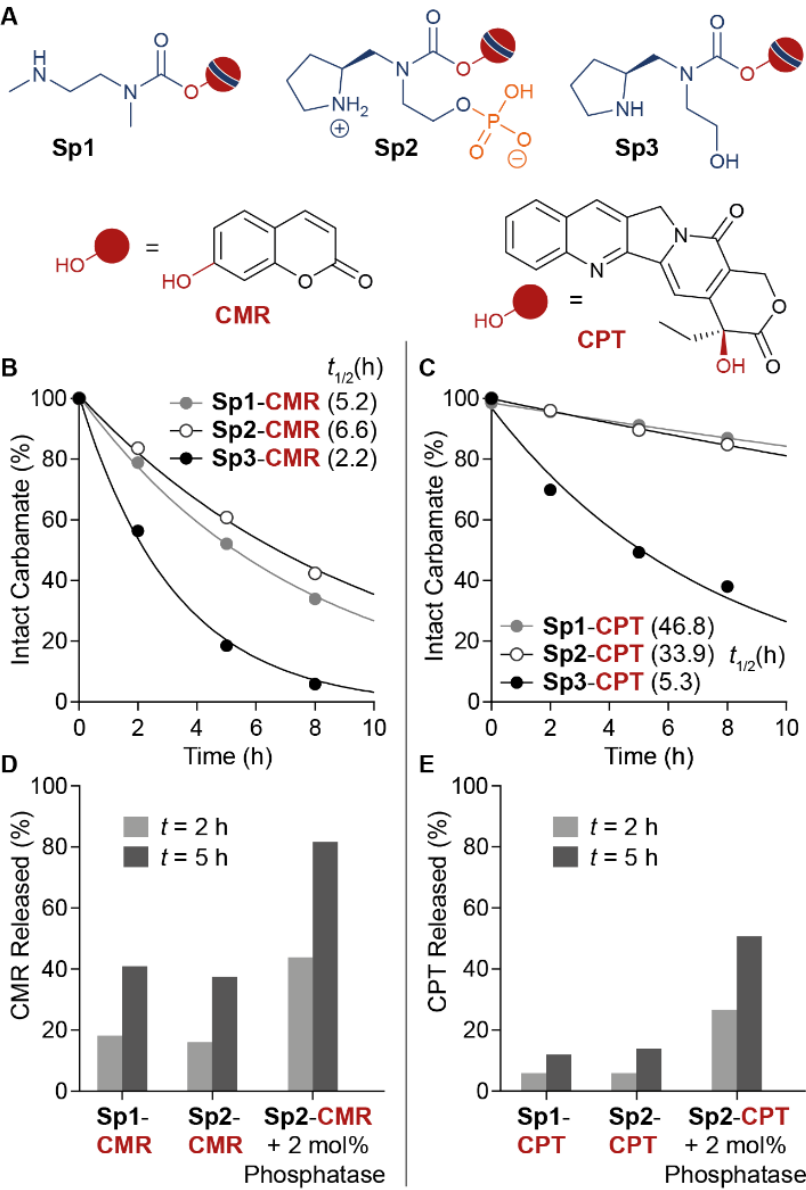

Figure 2. A) Structure of diamine-carbamate SI spacers Sp1-3, connected to either CMR or CPT. Summary of the HPLC data relative to the stability analysis of the six carbamates: release of CMR (B, 10\% DMSO solution in acetate buffer, $\mathrm{pH}$ 5.0) and release of CPT (C, $10 \%$ DMSO solution in HEPES buffer, $\mathrm{pH} 7.5$ ) at $37{ }^{\circ} \mathrm{C}$ (measured $t_{1 / 2}$ are reported in brackets). Evaluation of CMR (D) and CPT (E) release from SI spacer Sp2 in the presence/absence of phosphatase, in comparison with the standard ethylenediamine $\mathrm{S}$ spacer Sp1. Experimental procedures and HPLC traces are included in the ESI, + . HEPES = 4-(2-hydroxyethyl)-1-piperazineethanesulfonic acid.

As shown in Figure 1D, we selected phosphate monoesters as ideal $\mathrm{Br} \varnothing \mathrm{n} s t e d$ acids for this aim, owing not only to the low $\mathrm{p} K_{\mathrm{a}}$ values ( $\left.\mathrm{p} K_{\mathrm{a} 1}=1.54 ; \mathrm{p} K_{\mathrm{a} 2}=6.31\right),{ }^{12}$ but also to their susceptibility towards enzymatic cleavage (by phosphatases), which has been widely exploited in prodrugs ${ }^{13}$ as well as in other stimuliresponsive systems. ${ }^{14}$ To confirm that phosphate monoesters can inhibit the SI spacer degradation, we synthesized three different amine-carbamate SI spacers (Sp1-3 in Figure 2A, all synthetic procedures are reported in the ESI, + ) and compared the cyclization rates for the release of either the phenolic $\mathrm{OH}$ group of the fluorescent probe 7-hydroxycoumarin (CMR) or the tertiary $\mathrm{OH}$ of the anticancer drug camptothecin (CPT). As reported previously, ${ }^{11}$ Sp-CMR and Sp-CPT amine-carbamates were dissolved in a DMSO/aqueous buffer mixture at $\mathrm{pH} 5.0$ $(\mathrm{Sp}-\mathrm{CMR})^{15}$ or 7.5 (Sp-CPT) and incubated at $37{ }^{\circ} \mathrm{C}$. Aliquots were collected at different time points and the release of free CMR and CPT was monitored by HPLC (all UV traces are included in the $\mathrm{ESI},+)$, allowing the estimation of the spacers cyclization rates in terms of intact carbamate half-life $\left(t_{1 / 2}\right)$. As shown in Figure 2B-C, the phosphate-bearing Sp2 spacer showed low cyclization rates (Sp2-CMR $t_{1 / 2}=6.6 \mathrm{~h} ; \mathbf{S p 2 - C P T} t_{1 / 2}=34 \mathrm{~h}$ ). These half-lives were comparable to the ones shown by the ethylenediamine-carbamate Sp1 (Sp1-CMR $t_{1 / 2}=5.2 \mathrm{~h}$; Sp1-CPT $t_{1 / 2}=47 \mathrm{~h}$ ), a traditional bifunctional SI spacer whose poor efficacy has been described in different contexts. ${ }^{11,13 d, 16}$ Interestingly, the pyrrolidine-based spacer Sp3, devoid of the phosphate handle, showed the highest cyclization rates of the series (Sp3-CMR $t_{1 / 2}=2.2 \mathrm{~h}$; Sp3-CPT $t_{1 / 2}=5.3 \mathrm{~h}$ ). These data proved that the high reactivity of the pyrrolidine-carbamate $\mathrm{Sp} 3$ spacer towards cyclative $\mathrm{OH}$ release can be silenced by the presence of a proximal phosphate moiety. To confirm that the spacer reactivity can be restored by enzymatic dephosphorylation, we incubated amine-carbamates Sp2-CMR and Sp2-CPT alone or in the presence of a catalytic amount (2 mol\%) of phosphatase enzymes. Also in this case, the amount of released cargo was compared with the data relative to the analogous Sp1 derivatives. Figure 2D-E shows the significantly larger amount of free cargo liberated by the Sp2 aminecarbamates in the presence of phosphatase, as compared to Sp1 or Sp2 alone, which accounts for the rapid enzymatic conversion of Sp2 into the more efficient Sp3 spacer (HPLC traces relative to this analysis are included in the $E S I,+)$. Following these preliminary results, we implemented the trifunctional SI spacer in a full dual-activation system. In particular, the Sp2 and Sp3 spacers were used to connect the CPT hydroxyl group to a generic trigger (i.e. the Gly-Arg peptide, a known substrate for many proteases including trypsin), resulting in prodrugs 5 and $\mathbf{6}$ (Figure 3A). Similar to traditional release systems, the Gly-Arg proteolytic cleavage in $\mathbf{5}$ and $\mathbf{6}$ takes place at the dipeptide's $C$ terminus, which results in the rapid 1,6-elimination of a para-aminobenzyl carbamate spacer to give the azaquinone methide 7 , carbon dioxide and the amine-carbamates Sp2-CPT (from prodrug 5) and Sp3-CPT (from prodrug 6, Figure $2 \mathrm{~A}$ ). The stability of the prodrug 5 was analysed under different conditions, as shown in Figure 3B. In particular, a DMSO solution of $\mathbf{5}$ was added to a HEPES buffer solution at $\mathrm{pH} 7.5$ containing either the alkaline phosphatase enzyme or the protease trypsin or both of them, following incubation at $37^{\circ} \mathrm{C}$ and analysis by reverse-phase HPLC-MS. 
A
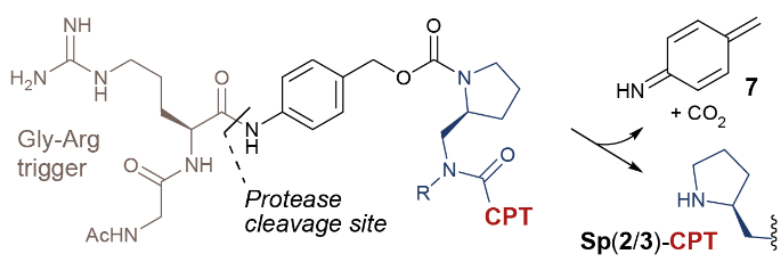

= Prodrug 5: Gly-Arg-Sp2-CPT; $\mathrm{R}=$ Prodrug 6: Gly-Arg-Sp3-CPT; $\mathrm{R}=$

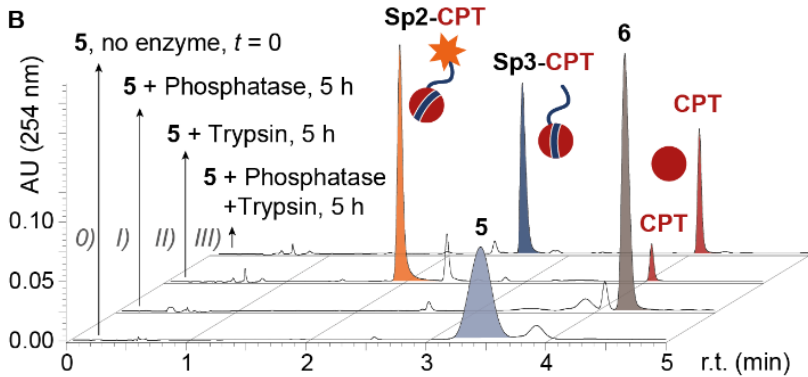

Figure 3. A) Molecular structure and trigger activation mechanism of protease-sensitive CPT prodrugs 5 and 6. B) HPLC traces relative to the stability analysis of prodrug $\mathbf{5}$ (2.5 $\mathrm{mM}$ in HEPES buffer at $\mathrm{pH} 7.5$ ) at $t=0$ (entry 0 ) or upon $5 \mathrm{~h}$ incubation at $37^{\circ} \mathrm{C}$ in the presence of $50 \mu \mathrm{M}$ alkaline phosphatase (entry I), $50 \mu \mathrm{M}$ trypsin (entry II) or $50 \mu \mathrm{M}$ alkaline phosphatase and $50 \mu \mathrm{m}$ trypsin (entry III). HPLC peaks assignment and integrations are reported in Figure S1 and S2 of the ESI, †. AU: Absorbance units; r.t.: retention time.

In this experiment, the UV peak corresponding to the intact $\mathbf{5}$ (see $t=0$ trace in Figure 3B, entry 0$)^{17}$ was not detectable after $5 \mathrm{~h}$ prodrug incubation in the presence of alkaline phosphatase (entry I), while a main peak was observed at longer retention times, identified as the dephosphorylation product (i.e. alcohol 6). On the contrary, treatment with trypsin (entry II) resulted in the full conversion of $\mathbf{5}$ into the Sp2-CPT amine-carbamate module. Under these conditions, a tiny amount of free CPT was detected, which is compatible with the slow cyclative cleavage of the Sp2 spacer. ${ }^{18}$ As compared to entry II, the $5 \mathrm{~h}$ exposure of 5 to both trypsin and phosphatase (entry III) led to a ca. 4fold larger amount of free CPT cargo. This observation reflects the cyclization half-life of the amine-carbamate Sp3-CPT $\left(t_{1 / 2}=\right.$ $5.3 \mathrm{~h}$, Figure $2 \mathrm{C}$ ), which was detected in the mixture as product of the two enzymatic degradations. Overall, this analysis confirmed not only that prodrug $\mathbf{5}$ is a substrate of both trypsin and alkaline phosphatase, but also that the action of both enzymes leads to a more efficient cargo release compared to the only peptide trigger activation.

Our data proved that para-aminobenzyl carbamate derivatisation of the pyrrolidine nitrogen inhibits the cargo release completely, whereas the phosphate group in the Sp2 spacer mediates a strong, yet partial inhibitory effect. While this aspect sets us the challenge of designing "perfect" nonsequential dual release systems, we investigated whether the inhibitory properties of the phosphate group in Sp2 are sufficient to elicit a detectable effect in a biological system. To this aim, we carried out tumour cell growth inhibition assays using prodrugs 5 and $\mathbf{6}$. In particular, the ovarian carcinoma cell line IGROV-1 was incubated for $72 \mathrm{~h}$ with the CPT prodrugs and free CPT, followed by quantitative analysis of cell growth (Table 1). As expected, prodrug 6 proved slightly less potent then free $\mathrm{CPT}\left(\mathrm{IC}_{50}=20\right.$ and $9 \mathrm{nM}$, respectively), and this difference is possibly due to $i$ ) the series of cleavage steps (i.e. peptide trigger activation and SI spacer cyclization) required for CPT release and ii) the different modes of cellular uptake of the free and derivatized CPT. ${ }^{[19]}$ Interestingly, the phosphate-bearing prodrug 5 proved the least active of the series ( $I_{50}=40 \mathrm{nM}$ ), which indicates that the phosphate group further inhibits CPT delivery. These data demonstrate that the kinetic effect on the CPT release provided by the phosphate group in prodrug $\mathbf{5}$ can influence the pharmacological effects also upon a long-term exposure of cancer cells to the prodrugs $(72 \mathrm{~h})$. This result, in line with our previous findings, ${ }^{11}$ supports the importance of a fast SI spacer cyclization in the drug release context, where the rapid accumulation of active payloads at the diseased site can prevent the development of drug resistance and increase the pharmacological effects. In a second experiment, we confirmed that the different cell growth activity shown by prodrugs $\mathbf{5}$ and 6 can be efficiently flattened by the addition of alkaline phosphatase to the cell medium (data shown in Figure S4).

Table 1. Cell growth-inhibition assays of IGROV-1 cells upon incubation with prodrugs 5 6 and free CPT. ${ }^{[a]}$

\begin{tabular}{ccc}
\hline Compound & & $\mathrm{IC}_{50}(\mathrm{nM})^{[\mathrm{b}]}$ \\
\hline CPT & Camptothecin & $9 \pm 2$ \\
Prodrug 5 & Gly-Arg-Sp2-CPT & $41 \pm 12$ \\
Prodrug 6 & Gly-Arg-Sp3-CPT & $20 \pm 2$ \\
\hline
\end{tabular}

[a] Assays were performed three times (three independent experiments) in duplicate. I $\mathrm{C}_{50}$ values were estimated by linear regression, as described in the ESI, + . [b] Cells were incubated for $72 \mathrm{~h}$ with serial dilutions of prodrugs 5-6 and free CPT.

In conclusion, this study indicates that the conjugation of a phosphate monoester group to an efficient amine-carbamate SI spacer can inhibit the cyclative carbamate cleavage for the release of $\mathrm{OH}$-bearing cargos. Notably, the original amine reactivity can be restored upon enzymatic hydrolysis of the phosphate group. We exploited this stimuli-responsive mechanism for the development of a dual-activation system, where the dephosphorylation of the trifunctional SI spacer can occur before or after the deprotection of the nucleophilic amine (i.e. the traditional "trigger activation" step, Figure 1A), resulting in a fast cargo release. To the best of our knowledge, this is the first example of "non-sequential dual activation" of a SI spacer. Unlike the "dual cargo functionalization" strategy (Figure 1C) our approach has a general application, since the trifunctional SI spacer is connected to a single anchoring point $(\mathrm{OH}$ group) at the cargo structure. Moreover, non-sequential dual activation strategies could represent a valid alternative to the wide range of sequential cascade approaches (Figure 1C), for instance whenever the actual order of activation signals in the given applicative context is unknown. Among its possible applications, our trifunctional SI spacer may be particularly suited to release highly cytotoxic drugs from tumour-targeting conjugates. First of all, activating enzymes such as proteases and phosphatases are often overexpressed in cancers, ${ }^{20}$ which holds promises for a selective drug release in the tumour mass. Moreover, the use of the phosphate-bearing Sp2 spacer represents a general strategy to reduce the hydrophobicity/enhance the hydrophilicity of tumour-targeting therapeutics (e.g. antibody-drug conjugates), which is an important feature to optimize pharmacokinetic profiles and therapeutic index. ${ }^{21}$ Finally, in the absence of phosphatases, our 
model prodrug $\mathbf{5}$ displayed a lower cell growth inhibitory activity than the phosphate-free analogue $\mathbf{6}$, even upon a longterm exposure $(72 \mathrm{~h})$ of cancer cells to the prodrugs. Conceivably, this protective effect, in the absence of Sp2 dephosphorylation, may be even more evident in vivo, since the highly hydrophilic Sp2-Drug metabolite (i.e. compound 2 in Figure 1D) should barely diffuse through cell membranes, while being rapidly cleared from the patient's body, reducing off-

\section{References}

1 A. Alouane, R. Labruère, T. Le Saux, F. Schmidt, L. Jullien, Angew. Chem. Int. Ed. 2015, 54, 7492.

2 a) S. Gnaim, D. Shabat, Acc. Chem. Res. 2014, 47, 2970; b) D. A. Roberts, B. S. Pilgrim, T. N. Dell, M. M. Stevens, Chem. Sci. 2020, 11, 3713; c) S. Davies, B. L. Oliveira, G. J. L. Bernardes, Org. Biomol. Chem. 2019, 17, 5725.

3 a) S. Huvelle, A. Alouane, T. Le Saux, L. Jullien, F. Schmidt, Org. Biomol. Chem. 2017, 15, 3435; b) Y. Meyer, J. A. Richard, B. Delest, P. Noack, P. Y. Renard, A. Romieu, Org. Biomol. Chem. 2010, 8, 1777; c) M. Ximenis, A. Sampedro, L. MartínezCrespo, G. Ramis, F. Orvay, A. Costa, C. Rotger, Chem. Commun. 2021, 57, 2736.

4 a) J. I. Scott, S. Gutkin, O. Green, E. J. Thompson, T. Kitamura, D. Shabat, M. Vendrell, Angew. Chem. Int. Ed. 2021, 60, 5699; b) S. Gutkin, O. Green, G. Raviv, D. Shabat, O. Portnoy, Bioconjugate Chem. 2020, 31, 2488; c) A. L. Acton, F. Leroux A. Feula, K. Melia, M. R. Sambrook, W. Hayes, A. T. Russell, Chem. Commun. 2019, 55, 5219.

5 a) Q. E. A. Sirianni, E. R. Gillies, Polymer, 2020, 202, 122638; b) T. N. Forder, P. G. Maschmeyer, H. Zeng, D. A. Roberts, Chem. Asian J. 2021, 16, 287.

6 a) Y. Xue, H. Bai, B. Peng, B. Fang, J. Baell, L. Li, W. Huang, N. H. Voelcker, Chem. Soc. Rev. 2021, 50, 4872; b) N. Krall, J. Scheuermann, D. Neri, Angew. Chem. Int. Ed. 2013, 52, 1384; c) R. Châtre, J. Lange, E. Péraudeau, P. Poinot, S. Lerondel, A. Le Pape, J. Clarhaut, B. Renoux, S. Papot, J. Controlled Release 2020, 327, 19.

7 a) S. Gnaim, D. Shabat, J. Am. Chem. Soc. 2017, 139, 10002; b) M. Grinda, J. Clarhaut, B. Renoux, I. Tranoy-Opalinski, S. Papot, Med. Chem. Commun. 2012, 3, 68; c) J. Tu, D. Svatunek, S. Parvez, H. J. Eckvahl, M. Xu, R. T. Peterson, K. N. Houk, R. M. Franzini, Chem. Sci. 2020, 11, 169.

8 a) F. Pretto, R. E. FitzGerald, Regul. Toxicol. Pharmacol. 2021, 122, 104890; b) A. Dal Corso, L. Pignataro, L. Belvisi, C. Gennari, Chem. Eur. J. 2019, 25, 14740.

9 H. G. Lerchen, B. Stelte-Ludwig, S. Berndt, A. Sommer, L. Dietz, A. S. Rebstock, S. Johannes, L. Marx, H. Jörißen, C. Mahlert, S. Greven, Chem. Eur. J. 2019, 25, 8208.

10 a) Initial photoactivation followed by hypoxic cleavage: $W$. Feng, C. Gao, W. Liu, H. Ren, C. Wang, K. Ge, S. Li, G. Zhou, H. Li, S. Wang, G. Jia, Z. Lia, J. Zhang, Chem. Commun. 2016, 52, 9434; b) initial activation with enzyme DT-diaphorase followed by photoactivation: Z. Chen, B. Li, X. Xie, F. Zeng, S. Wu, J. Mater. Chem. B 2018, 6, 2547; c) initial activation with target/systemic toxicity. ${ }^{22}$ Further activities are now in progress to improve the synthetic accessibility of phosphate-bearing SI spacers and to expand the toolbox of non-sequential activation strategies. We gratefully acknowledge Ministero dell'Università e della Ricerca (PRIN 2015 project 20157WW5EH) and Associazione Italiana per la Ricerca sul Cancro (grant number 24725) for financial support.

enzyme glucuronidase, followed by cathepsin B digestion: S. Chuprakov, A. O. Ogunkoya, R. M. Barfield, M. Bauzon, C. Hickle, Y. Cheol Kim, D. Yeo, F. Zhang, D. Rabuka, P. M. Drake, Bioconjugate Chem. 2021, 32, 746; d) initial activation with enzyme arylsulfatase $A$, followed by $\beta$-galactosidase digestion: J. D. Bargh, S. J. Walsh, N. Ashman, A. Isidro-Llobet, J. S. Carroll, D. R. Spring, Chem. Commun. 2021, 57, 3457.

11 A. Dal Corso, V. Borlandelli, C. Corno, P. Perego, L. Belvisi, L. Pignataro, C. Gennari, Angew. Chem. Int. Ed. 2020, 59, 4176.

12 T. S. Elliott, A. Slowey, Y. Ye, S. J. Conway, Med. Chem. Commun. 2012, 3, 735.

13 a) J. Rautio, N. A. Meanwell, L. Di, M. J. Hageman, Nat. Rev. Drug Disc. 2018, 17, 559; b) S. Kiran, Z. Hai, Z. Ding, L. Wang, Y. Liu, H. Zhang, G. Liang, Chem. Commun. 2018, 54, 1853; c) Q. Yao, F. Lin, X. Fan, Y. Wang, Y. Liu, Z. Liu, X. Jiang, P. R. Chen, Y. Gao, Nat. Commun. 2018, 9, 5032.

14 E. Procházková, P. Šimon, M. Straka, J. Filo, M. Májek, M. Cigáň, O. Baszczyňski, Chem. Commun. 2021, 57, 211.

15 Slightly acidic $\mathrm{pH}$ conditions were selected for our release analysis of Sp-CMR derivatives in order to facilitate the comparative analysis through HPLC methods. As a matter of fact, diamine-carbamate SI spacers release phenolic compounds too rapidly at higher $\mathrm{pH}$ values (see reference 11 ).

16 R. V. Kolakowski, K. T. Haelsig, K. K. Emmerton, C. I. Leiske, J. B. Miyamoto, J. H. Cochran, R. P. Lyon, P. D. Senter, S. C. Jeffrey, Angew. Chem. Int. Ed. 2016, 55, 7948.

17 Prodrug 5 proved stable for over $8 \mathrm{~h}$ in the absence of enzymes.

18 The CPT release through cyclization of the Sp2 and Sp3 spacers in entry $I I$ and III is confirmed by identification of corresponding cyclic ureas via MS analysis (see Figure S3 in the Supplementary Information).

19 R. V. J. Chari, M. L. Miller, W. C. Widdison, Angew. Chem. Int. Ed. 2014, 53, 3796.

20 a) O. Vasiljeva, D. R. Hostetter, S. J. Moore, M. B. Winter, Biol. Chem. 2019, 400, 965; b) A. Tomiyama, T. Kobayashi, K. Mori, K. Ichimura, Cancers 2019, 11, 241.

21 R. P. Lyon, T. D. Bovee, S. O. Doronina, P. J. Burke, J. H. Hunter, H. D. Neff-LaFord, M. Jonas, M. E. Anderson, J. R. Setter, P. D. Senter, Nat. Biotechnol. 2015, 33, 733.

22 The use of highly hydrophilic drugs is a well-known approach to reduce off-target toxicity in tumor-targeting conjugates, see: A. Maderna, C. A. Leverett, Mol. Pharmaceutics 2015, 12 , 1798. 